International Journal of Modern Physics B,

(C) World Scientific Publishing Company

\title{
POLARON CRYSTALLIZATION AND MELTING: EFFECTS OF THE LONG-RANGE COULOMB FORCES
}

\author{
S. FRATINI and P. QUÉMERAIS \\ Laboratoire d'Etudes des Propriétés Electroniques des Solides, CNRS \\ BP 166, 38042 Grenoble Cedex 9, France
}

Received 30 October 1998

Revised

\begin{abstract}
On examining the stability of a Wigner crystal in an ionic dielectric, two competitive effects due to the polaron formation are found to be important: (i) the screening of the Coulomb force $1 / \varepsilon_{s} r$, which destabilizes the crystal, compensated by (ii) the increase of the carrier mass (polaron mass). The competition between the two effects is carefully studied, and the quantum melting of the polaronic Wigner crystal is examined by varying the density at zero temperature. By calculating the quantum fluctuations of both the electron and the polarization, we show that there is a competition between the dissociation of the polarons at the insulator-to-metal transition (IMT), and a melting towards a polaron liquid. We find that at strong coupling, a liquid state of dielectric polarons cannot exist, and the IMT is driven by the polaron dissociation. Next, taking into account the dipolar interactions between localized carriers, we show that these are responsible for an instability of the transverse vibrational modes of the polaronic Wigner crystal as the density increases. This provides a new mechanism for the IMT in doped dielectrics, which yields interesting dielectric properties below and beyond the transition. An optical signature of such a mechanism for the IMT is provided.
\end{abstract}

\section{Introduction}

In a recent letter to Nature, Phillips et al. 1 pointed out that a superconducting state could be expected close to the instability of a 2D Wigner Crystal (WC) of electrons. Previously, Takadal had mentioned such a possibility for a 3D WC. In both cases, the pairing mechanism is due to correlation between electrons, mediated by the long range Coulomb interaction. More explicitely, pairing occurs through the Coulomb holes which form in the vicinity of the melting transition. Translated into the language of dielectric constants, as was first recognized by Bagchil for the $3 \mathrm{D} \mathrm{WC}$, it means that the static dielectric constant is negative. In his original paper, Bagchi also extended his results to an insulating lattice of Lorentz dipoles. He found that, on increasing the dipole density, the Lorentz lattice undergoes a phonon instability due to the dipolar interactions. At the same time, the static dielectric constant becomes identical to the dielectric constant of an ordinary WC, and thus can be negative. In other words, an ordinary WC appears to be nothing but a Lorentz lattice of dipoles just on the verge of instability. This could provide a natural pairing mechanism for (hypothetical) free electrons moving through such a Lorentz lattice close to its critical point. Quite generally, the question of the 
sign of the static dielectric constants has been addressed by many authors. Cohen and Anderson 1 argued on physical grounds that such a negative sign would be impossible. Later, the "russian school", in particular Dolgov et al. ., following the previous work of Martin6, pointed out that a negative sign of the dielectric constant would not contradict any stability criterion. The only required condition is that $\varepsilon(0,0)$ be strictly positive: a system can have $\varepsilon(\mathbf{k}, 0)<0$ for any $k>0$, and still be stable.

We have developed 1 such a physical picture in order to describe the Insulatorto-Metal transition in ionic dielectrics, taking into account the formation of large polarons when introducing the doping charges. These polarons, which are ordered in a crystallized state at low densities, behave more or less as Lorentz dipoles, and can yield some of the interesting physical properties already mentioned by Bagchi. In order to understand our point of view, it is useful to connect our studies to the recent "fight" in Physical Review Letters of Chakraverty et al. against the (bi)polaronic superconductivity scenario 10 . These authors did not find the bipolaronic scenario suitable for the interpretation of the experimental data in highTc, a point of view which seems to be fully shared by Andersong. In fact, we would like to underline that the basic building block of the (bi)polaronic scenario is the existence of a quantum liquid of (bi)polarons. As we will explain later, this turns out to be impossible at strong electron-phonon coupling, owing to the drastic role of long-range Coulomb forces in the many-polaron problem. Indeed, treating self-consistently a finite density of interacting polarons is a much more complicated problem than considering only one or two polarons. Extensions to finite densities usually neglect the Coulomb interaction between (bi)polarons, as in the framework of the random phase approximation 10 . As we will see, this approximation is quite dubious at strong polar electron-phonon coupling, while it should remain reasonable at small and intermediate coupling11. Long-range Coulomb forces are responsible for both the polaron formation (if one only considers the coupling of the electrons to the polar mode of the crystal, as it is the case in all our studies), and for their mutual interactions. In such systems, electron-phonon and electronelectron interactions cannot be decoupled and must be treated on the same ground, otherwise the collective behavior of polarons at finite density can be completely missed. To tackle the many-polaron problem, we have considered a system at low density. Starting from the remark 33 that two polarons at a distance $d$ repel as $1 / \varepsilon_{s} d$ if the dielectric constants satisfy $\varepsilon_{\infty} / \varepsilon_{s}>\approx 0.1$ (as it is well-known12, there is no bipolaron formation in that case), at low densities, the crystallized state is necessarily the ground-state.

Let us consider a WC, consisting of a system of electrons plus a jellium (assumed to be rigid) of positive charge with the same density. We can imagine that this WC is inserted into a host polar material, characterized by the two limiting dielectric constants $\varepsilon_{s}, \varepsilon_{\infty}$ and by the frequency of the longitudinal optical mode $\omega_{L O}$. If one turns on the coupling between the WC and the polar phonon mode of the host material, two competitive effects arise. The first one corresponds to the screening 
of the Coulomb interaction between the electrons, which is reduced from $1 / \varepsilon_{\infty} r$ to $1 / \varepsilon_{s} r$. This screening is due to the host lattice polarization which surrounds the electrons, which is nothing but the polaron cloud. The other important effect is the increase of the carrier mass: when the coupling with the lattice is turned on, the original band mass $m^{*}$ becomes $M_{P}$, the polaron mass, which can be large, especially in the strong coupling limit for which the polarons are well-formed. Now, the crystallization is due to the overcoming of the kinetic energy $1 / M_{p} R_{s}^{2}$ by the Coulomb repulsion $1 / \varepsilon_{s} R_{s}$, where $R_{s}$ is related to the density $n$ as usual: $n=$ $3 / 4 \pi R_{s}^{3}$. We see from this simple argument that the polaronic crystallized state can be stable up to fairly high densities in spite of the screening of the Coulomb forces. Furthermore, as given by standard polaron theory, the mass $M_{P}$ becomes infinite as the phonon frequency tends to zero. In that case, i.e. when $\omega_{L O} \rightarrow 0$, the kinetic energy is always negligible with respect to the Coulomb repulsion, and melting towards a liquid state of polarons is clearly not possible.

In our studies, we have focused on the quantum melting (at zero temperature) of such a polaronic Wigner crystal (PC). We have found that the two competitive effects above give rise to two different scenarios for the IMT, which can be easily understood. When the electron-phonon coupling vanishes, we are left with an ordinary WC of electrons. As the density is raised, the quantum fluctuations of the localized particles increase, and when they reach a certain magnitude, the crystal melts towards a liquid state of electrons. The effect of the host lattice is negligible in that case. In the opposite limit $\omega_{L O} \rightarrow 0$, the electron-phonon cort pling becomes infinite. Its strength is given by the usual dimensionless parameter 4 $\alpha=\left(m^{*} / 2 \hbar^{3} \omega_{L O}\right)^{1 / 2} e^{2} / \tilde{\varepsilon}$, where $1 / \tilde{\varepsilon}=1 / \varepsilon_{\infty}-1 / \varepsilon_{s}$ is the effective dielectric constant responsible for the polaron formation. In this limit, each electron is selftrapped in a polarization potential-well which is frozen. As it is well-known, the latter has a coulombic nature and behaves as $1 / \tilde{\varepsilon} r$ at large distance. Since the polarization cannot move, the polarons, i.e. the electron plus the polarization, cannot go towards a liquid state. The IMT is thus driven by the dissocation of the polarons and the screening of the polarization potentials by the liberated electrons. We are in a situation which closely resembles the usual Mott transition. On the basis of the same argument as Mott国, the critical density can be estimated to be:

$$
n_{c}^{1 / 3}\left(\tilde{\varepsilon} / \varepsilon_{\infty}\right) R_{P} \approx 0.25
$$

where $R_{P}$ is the bound-state radius, which is nothing but the polaron radius given at strong coupling by $R_{P} \approx 3.2 \tilde{\varepsilon}\left(m^{*} / m_{e}\right) a_{0}$. Here, $a_{0}$ is the usual Bohr radius $(0.53 \AA)$, and $R_{P}$ corresponds to a hydrogenic wave-function.

Starting from the limiting cases presented above, it is now important to extend the results to any finite electron-phonon coupling (i.e. to any finite phonon frequency), and examine which mechanism is responsible for the melting of the polaron crystal. At this stage of the theory, we have developed two steps. 

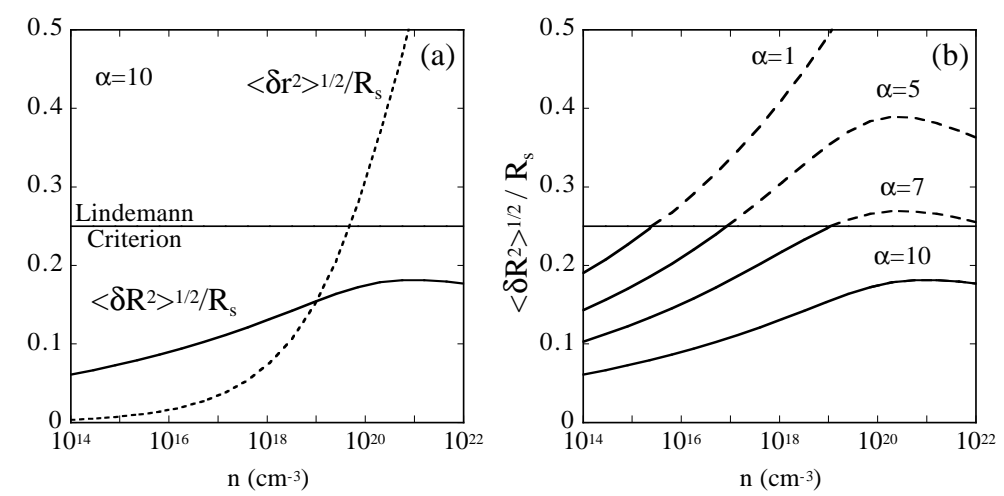

Figure 1: (a) The fluctuation of the polaron $\left\langle\delta R^{2}\right\rangle^{1 / 2} / R_{s}$ (bold line) and of its internal degree of freedom $\left\langle\delta r^{2}\right\rangle^{1 / 2} / R_{s}$ (dotted line) versus $n$ at $\alpha=10$. (b) The polaron fluctuation $\left\langle\delta R^{2}\right\rangle^{1 / 2} / R_{s}$ versus $n$ for different $\alpha$. Physical parameters are $\epsilon_{\infty}=5, \epsilon_{s}=30$ and $m^{*}=2 m_{e}$. The density parameter is simply $n=1.6 \cdot 10^{24} / r_{s}^{3}$.

\section{Quantum melting: polaron breaking versus polaron liquid}

First, we have modeled the situation in the PC by considering a single polaron localized in a positively charged jellium sphere with density $n=3 / 4 \pi R_{s}^{3}$. In this approach, which is a sort of mean-field many-body calculation (see for example the first papers by Wigner on the electron crystallization), dipolar interactions and exchange effects are neglected. However, one obtains reliable results on the crystal energy and ground state properties, provided the density is low enough (large $\left.R_{s}\right)$. As it was done by Nozieres et al. for the WCE, we have applied the Lindemann criterion to this model. These calculations allow us to understand, at least qualitatively, the route that the crystal will choose to melt: polaron dissociation versus polaron liquid state. Of course, this method just gives access to the melting scenario, not to the ground-state beyond the transition.

The application of this criterion to the PC is more complicated than for an ordinary WC, owing to the composite nature of the polarons. To be able to delocalize the polarons towards a liquid state, the quantum fluctuations for each polaron moving as a whole, i.e. the motion of each electron together with its surrounding polarization, must become large in comparison with $R_{s}$. On the contrary, if the relative fluctuations of the electron with respect to the polarization become large, the polarons break apart (dissociation). The Feynman treatment for the polaron 17 , which relies on path integral calculations, yields a natural way to evaluate such 
quantities: since the polarization field in Feynman's approach is replaced by a rigid particle with coordinate $X$, one easily calculates both the quantum fluctuations of the center of mass $R=\left(m^{*} x+M X\right) /\left(m^{*}+M\right)$ and the relative coordinate $r=x-X$ ( $x$ is the electron coordinate). Thus, we now have two different Lindemann criteria, which correspond to two different melting scenarios:

(i) $\left\langle\delta R^{2}\right\rangle^{1 / 2} / R_{s}>1 / 4$ which allows the melting of the crystal towards a liquid state of polarons;

(ii) $\left\langle\delta r^{2}\right\rangle^{1 / 2} / R_{s}>1 / 4$ for the polaron dissociation.

Both quantities are calculated by the same path integral variational procedured t 7 .

The frequencies of the two degrees of freedom $r$ and $R$, respectively $\omega_{\text {int }}$ and $\omega_{\text {ext }}$, were calculated in ref.], together with the ratios (i) and (ii) (see Fig.1) for different $\alpha$. From the basic observation that a polaron is a bound state of an electron plus a phonon cloud, it was pointed out that the frequency of vibration of the polaron as a whole is physically limited by the phonon frequency $\left(\omega_{\text {ext }} \leq \omega_{L O}\right)$. In other words, at strong electron-phonon coupling (or equivalently small $\omega_{L O}$ ), the lattice polarization cannot dynamically follow the increase in kinetic energy induced by the doping, and the quantum fluctuations are transferred to the internal degree of freedom, breaking the polaron apart. For that reason, the polarons are expected to dissociate at the transition for sufficiently high $\alpha\left(\alpha>\alpha^{*} \simeq 7.5\right)$, rather than melting to a polaron liquid.

We have also calculated the same quantities for an anisotropic polaron. By anisotropic, we mean that the band mass of the electron is different in the $x y$ plane than in the $z$ direction, by means of the phenomenological parameter $b=m_{x y} / m_{z}$. However, the polarization is assumed to preserve its three-dimensional character. This is the case for cuprates, as we have already dicussed , on the basis of Chen et al. experiments 18 . When $b=1$, we recover the usual 3D polaron, while for $b=0$, we have some kind of $2 \mathrm{D}$ polaron (the electron motion is restrained to the $x y$ plane). Table 1 summarizes the numerical results obtained for two different band masses $m^{*}=m_{e}$ and $m^{*}=2 m_{e}$, for both $b=1$ and $b=0$.

\begin{tabular}{l|ccc}
\hline \hline & $\alpha^{*}$ & $n_{c}\left(m^{*}=m_{e}\right)$ & $n_{c}\left(m^{*}=2 m_{e}\right)$ \\
\hline isotropic $(b=1)$ & 7.5 & $6 \cdot 10^{18}$ & $5 \cdot 10^{19}$ \\
(fully) anisotropic $(b=0)$ & 4.4 & $8 \cdot 10^{19}$ & $6 \cdot 10^{20}$ \\
\hline \hline
\end{tabular}

Table I. Critical density $n_{c}\left(\mathrm{~cm}^{-3}\right)$ and coupling $\alpha^{*}$ (see text)

The important qualitative fact that we can conclude from the numerical data is the following: what happens for $\alpha>7$ in 3D, will happen for lower couplings $(\alpha>4)$ in the anisotropic case. In other words, the polaron dissociation may be a physical possibility in the cuprates for describing the IMT mechanism. at view of the fairly high value of the polar electron-phonon coupling (Chen et al 18 estimated $\alpha \approx 5$ for $\mathrm{La}_{2} \mathrm{CuO}_{4}$ ). 


\section{Effect of the dipolar interactions: Bagchi's instability}

Let us now concentrate on the melting in the 3D case, and restrain ourselves to the limit $\alpha \rightarrow \infty\left(\omega_{L O} \rightarrow 0\right)$. In this regime, the lattice polarization around each electron is frozen, and we can easily include the dipolar interactions that were previously neglected. The electrons vibrate in a potential-well due to the static polarization, the jellium sphere and the electrons on the other sites. Assuming that these are localized on the sites of a Bravais lattice, the crystal of polarons can be viewed as a Lorentz lattice of dipoles. Each individual dipole vibrates at the frequency $\omega_{\text {int }}$ above mentioned. Bagchil 3 has already calculated (classically) the phonon dispersion and the dielectric properties of such a Lorentz lattice. It can be shown 3 that the vibrational spectrum $\Omega(\mathbf{k}, \lambda)$ of such a lattice ( $\mathbf{k}$ is the wave vector and $\lambda=1,2,3$ is the phonon branch index) satisfies the following relations:

$$
\Omega^{2}(\mathbf{k}, \lambda)=\omega^{2}(\mathbf{k}, \lambda)+\omega_{p o l}^{2},
$$

where we have defined $\omega_{\text {pol }}^{2}=\omega_{\text {int }}^{2}-\omega_{p}^{2} / 3 \varepsilon_{\infty}, \omega_{p}^{2}=4 \pi n e^{2} / m^{*}$ being the usual plasma frequency (defined without any dielectric constant). $\omega(\mathbf{k}, \lambda)$ in (2) is nothing but the phonon spectrum of a usual WC. It satifies the Kohn sum rule3: $\sum_{\lambda=1}^{3} \omega(\mathbf{k}, \lambda)^{2}=$ $\omega_{p}^{2} / \varepsilon_{\infty}$. At long wavelengths, there is one optical mode $\left(\omega(0\right.$, opt. $\left.)=\omega_{p} / \sqrt{\varepsilon_{\infty}}\right)$ and two acoustical modes. From these properties of $\omega(\mathbf{k}, \lambda)$ and using (2), it comes out immediately that the long wavelength transverse modes of the polaron crystal become unstable when $\omega_{\text {pol }}^{2}<0$, i.e. when $\omega_{\text {int }}<\omega_{p} / \sqrt{3 \varepsilon_{\infty}}$. The critical density for which $\omega_{\text {pol }}\left(n_{c}\right)=0$, based upon the calculation of $\omega_{\text {int }}$ in section 2 , is $n_{c}^{\text {Bag. }} \approx 5 \cdot 10^{20} \mathrm{~cm}^{-3}$ for $m^{*}=2 m_{e}, \varepsilon_{s}=30$ and $\varepsilon_{\infty}=5$, which is slightly higher than the value obtained through the Lindemann criterion.

The (longitudinal) dielectric constant $\varepsilon(\mathbf{k}, \omega)$ of the Lorentz lattice has also been calculated by Bagchi.

$$
\frac{1}{\varepsilon(\mathbf{k}, \omega)}=\frac{1}{\varepsilon_{\infty}}\left[1-\frac{\omega_{p}^{2} / \varepsilon_{\infty}}{k^{2}} \sum_{\lambda} \frac{\left(\mathbf{k} \cdot \mathbf{e}_{\mathbf{k}, \lambda}\right)^{2}}{\left[\omega^{2}(\mathbf{k}, \lambda)+\omega_{p o l}^{2}-\omega^{2}\right]}\right]
$$

where $\mathbf{e}_{\mathbf{k}, \lambda}$ is the polarization wavevector of the phonon mode $\mathbf{k}, \lambda$. It can be easily evaluated both in the static and in the long wavelength limit. In the latter case, we have:

$$
\varepsilon(0, \omega)=\varepsilon_{\infty}\left[1-\frac{\omega_{p}^{2} / \varepsilon_{\infty}}{\omega^{2}-\omega_{p o l}^{2}}\right],
$$

which is sketched in Fig.2.a. The pole in the dielectric function, located at $\omega_{\text {pol }}$ (i.e. the polaron peak in the optical conductivity), is shifted to lower energies as the density increases, and tends to zero on approaching the IMT, as shown in Fig.2.b. Strictly speaking, since $\omega_{L O}$ is never zero, but assumed to be small, $\omega_{\text {pol }}$ should rather saturate at higher density. The corresponding behavior of the optical conductivity $\sigma(\omega)=\operatorname{Im}[\varepsilon(0, \omega)] \omega / 4 \pi$ and energy loss function $\operatorname{Im}[-1 / \varepsilon(0, \omega)]$ at $T=0$ is sketched in figure 3 . The polaronic peak shift seems to have been recently 

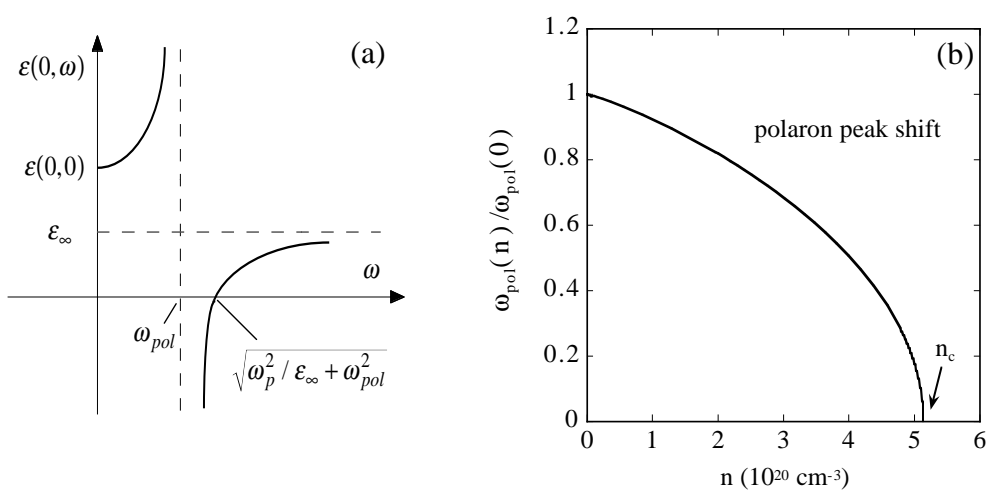

Figure 2: (a) sketch diagram of the long wavelength dielectric function, (b) shift of the polaron peak and crystal instability in the static limit $\left(\omega_{L O}=0\right)$, for $m^{*}=2 m_{e}$.

observed 19 in the cuprates. Even though our calculations are strictly valid only in the limit $\omega_{L O} \rightarrow 0$, one can however argue that for $\alpha>\alpha^{*}$ (above mentioned), the results - in particular the existence of Bagchi's instability - remain qualitatively correct, since in that case the polarization cannot easily follow the electron vibrations. New kinds of collective modes 21 corresponding to the mixture of polarization waves with electron vibrations can also be expected, as was pointed out by Bozovic22, which should have an optical signature.

As mentioned in the introduction, the static dielectric constant also exhibits interesting properties in this limit. From (3), it can be seen that at the critical density $\left(\omega_{\text {pol }}=0\right)$, owing to the sum rule, the phonon dispersion obeys $\omega(\mathbf{k}, \lambda)<\omega_{p} / \sqrt{\varepsilon_{\infty}}$. Therefore, we necessarily have $\varepsilon(\mathbf{k}, 0)<0$ for any $k>0$. This indicates the possibility of having a net attraction between free carriers coexisting with localized polarons beyond the transition. This possibility opens up very interesting questions for both a superconducting scenario, and unusual properties of the normal phase beyond the IMT. Pseudo-separation between localized (heavy) polarons and free (light) electrons has already been mentioned 7.23.23.24 both theoretically and experimentally. We believe that Bagchi's instability can be one of the keys of this pseudo-separation. Evidently, at the critical density, some polarons must be destroyed. However, it is not necessary to destroy all the polaron lattice, and we just need to fulfill the stability condition on the vibrational modes $\Omega^{2}$ ( $k$,trans.) $\geq 0$ (i.e. $n_{\text {polarons }} \leq n_{c}^{\text {Bag. }}$ ). On the other hand, there is some energetical argument in favor of a mixed phase of localized polarons with free electrons. The polaron 

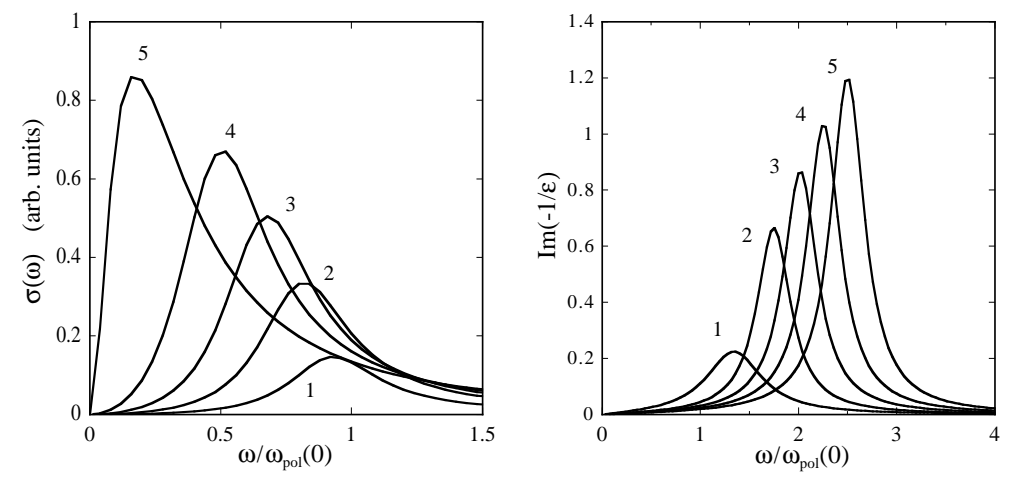

Figure 3: (a) optical conductivity and (b) EELS spectrum, as calculated by introducing an arbitrary Lorentzian broadening in eq.(4). Labels 1-5 stand for the density in units of $10^{20} \mathrm{~cm}^{-3}$. Physical parameters are the same as in Fig.1, and $n_{c} \approx 5 \cdot 10^{20} \mathrm{~cm}^{-3}$.

formation energy being negative, it costs energy to destroy them. A compromise might be achieved (which remains to be proved) by the coexistence of a polaron lattice with density $n_{c}^{B a g}$, and quasi-free electrons, provided the mean distance between free electrons (corresponding roughly to the screening length) is larger than the distance between localized polarons. However, above a certain density (say $n_{\text {free }} \approx n_{c}^{\text {Bag. }}$, all the polarons must disappear due to a global screening effect. This last situation could correspond to the overdoped region in the cuprates, while the coexistence between localized polaron and free electrons, to the underdoped region.

\section{Conclusion}

The studies proposed in this paper (and previous references) are currently being extended in several directions, essentially at finite temperature and finite phonon frequency, including the dipolar interactions 20 . The possibility of a mixed state of crystallized polarons coexisting with quasi-free carriers is important and must be clarified. Another problem concerns the possibility of having a crystal of bipolarons instead of polarons. This is based on the recent results of Moulopoulos and Ashcroft 25 for the ordinary WC, where they have proved that at intermediate densities, a paired electron crystal is more stable than the single electron crystal. In the polaronic case, this is still an open question. Nevertheless, it would not change the 
basic result of our studies: at strong coupling, the IMT is driven by the dissociation of some localized (bi)polarons and cannot yield a liquid state of (bi)polarons.

In conclusion, let us underline that what we have called the Bagchi instability, can also be supported by the existence of localized dipoles which have nothing to do with polarons. This remark comes in mind if one considers another family of doped oxides, the bismuthates $\left(K_{x} \mathrm{Ba}_{1-x} \mathrm{BiO}_{3}\right)$, where it has been recently suggested that dipolar interactions could be of importance 26 .

Part of this work has been presented at the International Conference STRIPES in Rome (2-4 june 1998), and took its origin from very long discussions that one of us (P.Q.) had with A. Bianconi during the year 1993, on the possible crystallization of polarons in the cuprates. This work received financial support from the European Commission (contract no. ERBFMBICT 961230).

\section{References}

1. P. Phillips, Y. Wan, I. Martin, S. Knysh, D. Dalidovich, Nature, Vol. 395, p.253 (1998)

2. Y. Takada, Phys. Rev. B, 47, p.5202 (1993)

3. A. Bagchi, Phys. Rev. 178, 3, p.707 (1969)

4. M.L. Cohen, P.W. Anderson, "comments on the maximum superconducting transition temperature", in Superconductivity in $d$ - and f-band metals, ed. by D.H. Douglass (AIP, New-York), p.17 (1972)

5. O.V. Dolgov, D.A. Kirzhnits, E.G. Maksimov,Rev. Mod. Phys., 53, p.81 (1981)

6. P. Martin, Phys. Rev., 161, p.143, (1967)

7. P. Quémerais, Mod. Phys. Lett. B 9, 25, p. 1665 (1995); P. Quémerais and S. Fratini, Mod. Phys. Lett. B 11, 30, p. 1303 (1997), cond-mat/9709182; P. Quémerais and S. Fratini, Proceedings of New3SC-1, Baton-Rouge (February 1998), to be published in Int. J. Mod. Phys. B.

8. B.K. Chakraverty, J. Ranninger, D. Feinberg, Phys. Rev. Lett., 81, p. 433, (1998) ; A.S. Alexandrov, Comment on Chakraverty et al. letter, ; Chakraverty et al. Reply

9. P.W. Anderson, Phys. Rev. Lett., 79, p.4717, (1997)

10. A.S. Alexandrov and N.F. Mott, Rep. Prog. Phys. 57, p.1197 (1994)

11. G. Iadonisi, G. Capone, V. Cataudella, G. de Filippis, Phys. Rev. B 53, p.13497 (1996) ; G. de Filippis, V. Cataudella, G. Iadonisi, preprint cond-mat/9810365, to be published in Eur.Phys.Jour.(1998).

12. G. Verbist, M.A. Smondyrev, F. M. Peeters, and J. T. Devreese, Phys. Rev. B 45, p.5262 (1992) and references therein

13. A.A. Remova, B. Ya. Shapiro, Physica C, 160, p.202 (1989)

14. H. Fröhlich, Adv. in Phys. 3, p.325 (1954); C.G. Kuper and G.D. Whitfield, Polarons and excitons, (Oliver and Boyd, Edinburgh and London, 1962)

15. N.F. Mott, Phil. Mag. 6, p.2897 (1961)

16. P. Nozières and D. Pines, Phys. Rev. 111, 2, p.442 (1958)

17. R. P. Feynman, Phys. Rev. 97, 3, p.660, (1955)

18. C.Y. Chen, R.J. Birgeneau, M.A. Kastner, N.W. Preyer and T. Thio, Phys. Rev., B 43, p.392 (1991)

19. B. Ruzicka, S. Lupi, P. Calvani, M. Capizzi, P. Maselli, Physica B 244, p.41, (1998) and P. Calvani, private communication

20. S. Fratini, P. Quemerais, in preparation

21. N.W. Ashcroft, private communication 
22. I. Bozovic, Phys. Rev. B 48, p.876 (1993)

23. A. Bianconi, Proceed. of the Int. Conf. on Materials and mechanism of Superconductivity High Temperature Superconductors IV, Grenoble (1994), ed. P. Wyder (North-Holland), p. 269, and ref. therein.

24. D. Mihailovic and K.A. Müller, Proc. NATO ASI "Materials aspects of High-Tc Superconductivity: 10 years after the discovery", Kluwer, London (1997), p.243 ; K.A. Muller, G. Zhao, K. Conder, H. Keller, J.Phys.Cond.Mat., 10, L291 (1998)

25. K. Moulopoulos, N.W. Ashcroft, Phys. Rev. Lett. 69, 17, p.2555 (1992); Phys. Rev. $B$ 48, 16, p.11646 (1993)

26. V.G. Orlov, Int. Conf. on Strongly Correlated Electron Systems, Paris, 15-18 June 1998, abstracts 